https://doi.org/10.22319/rmcp.v9i3.4440

Artículo

\title{
Evaluación del rendimiento de materia seca y sus componentes en germoplasma de alfalfa (Medicago sativa $L$.)
}

\section{Performance of dry matter yield and its components in germoplasm of alfalfa (Medicago sativa $L$.)}

Milton Javier Luna Guerrero ${ }^{\mathrm{a}}$

Cándido López Castañeda $\mathrm{b}^{*}$

Alfonso Hernández Garay ${ }^{\mathrm{a}, \dagger}$

Pedro Arturo Martínez Hernández ${ }^{\mathrm{c}}$

María Esther Ortega Cerrilla ${ }^{a}$

${ }^{a}$ Postgrado en Recursos Genéticos y Productividad-Genética, Colegio de Postgraduados. Carretera México-Texcoco km 36.5, Montecillo, Texcoco, Estado de México, México.

${ }^{\mathrm{b}}$ Postgrado en Producción Animal, Universidad Autónoma Chapingo. Chapingo, México.

C Postgrado en Producción Animal, Universidad Autónoma Chapingo. Chapingo, México.

* Autor de correspondencia: clc@colpos.mx.

\section{- Resumen:}

Se evaluó el comportamiento productivo en 100 familias de medios hermanos (FMH) derivadas de las variedades San Miguel y Oaxaca, y las poblaciones San Miguel x Oaxaca y Oaxaca x San Miguel, en macetas a la intemperie en Montecillo, Texcoco, Estado de México. Se realizaron cortes cada cinco semanas en otoño-invierno 2013-2014 y cada cuatro semanas en primavera 2014. Las FMH derivadas de San Miguel x Oaxaca y Oaxaca x San Miguel tuvieron en promedio $10 \%$ mayor rendimiento de materia seca (RMS), tasa absoluta de crecimiento (TAC) y relación hoja:tallo (H:T) por planta, que las FMH derivadas de San Miguel y Oaxaca, en cada corte y estación del año. Las FMH derivadas de San Miguel presentaron mayor número de tallos (NT) en invierno y primavera, y menor altura de planta (AP) en primavera que las de Oaxaca y las FMH derivadas de San Miguel x Oaxaca y Oaxaca 
x San Miguel. El RMS y TAC en primavera fueron 57 y $33 \%$ más altos que en otoño e invierno. La relación H:T en otoño fue $5 \%$ mayor que en invierno y $30 \%$ mayor que en primavera; el mayor NT y AP se presentaron en primavera. La eficiencia en el uso de la radiación (EUR) fue mayor en primavera que en otoño e invierno con los valores más altos en abril. La variabilidad entre las FMH derivadas de San Miguel x Oaxaca y Oaxaca x San Miguel, indica la posibilidad de seleccionar familias superiores en rendimiento de materia seca.

- Palabras clave: Rendimiento estacional, Rendimiento por corte, Relación hoja:tallo, Eficiencia radiación.

\begin{abstract}
- Abstract:
The present research work was carried on to study the productive performance of a group of 100 half-sib families (HSF) derived from each of Oaxaca and San Miguel commercial varieties, and San Miguel x Oaxaca and Oaxaca x San Miguel populations, in pots placed outdoors at Colegio de Postgraduados, Montecillo, Texcoco, State of Mexico. Harvests of aerial biomass were made every 5 wk during the fall-winter growing season 2013-2014 and 4 wk during the spring growing season 2014. The HSF derived from San Miguel x Oaxaca and Oaxaca $x$ San Miguel had $10 \%$ higher dry matter yield (DMY), absolute crop growth rate (ACGR) and leaf:stem ratio (L:S) per plant than the HSF derived from San Miguel and Oaxaca in every growing season and harvest. On the other hand, the HSF derived from San Miguel had greater number of tillers (NS) per plant in the winter and spring seasons, and lower plant height $(\mathrm{PH})$ in the spring season than the HSF derived from Oaxaca, San Miguel $x$ Oaxaca and Oaxaca x San Miguel. The DMY and ACGR were $57 \%$ and $33 \%$ higher in spring than in fall and winter. The L:S was $5 \%$ higher in fall than in winter and $30 \%$ higher than in spring; the greatest values for NS and $\mathrm{PH}$ were measured in spring. The radiation use efficiency (RUE) had higher values in spring than in fall and winter with the highest values in April. The observed variability among the HSF derived from San Miguel x Oaxaca and Oaxaca x San Miguel, suggests the possibility of selecting families with higher dry matter yield.
\end{abstract}

- Key words: Dry matter, Herbage yield, Leaf:Stem ratio, Radiation efficiency.

Recibido 19/03/2017.

Aceptado 23/10/2017. 


\section{Introducción}

La alfalfa (Medicago sativa L.) es un cultivo perenne de importancia mundial, debido a su alta calidad nutritiva, alto rendimiento, amplia adaptación, persistencia y capacidad de asociarse en simbiosis con bacterias del género Rhizobium, que fijan nitrógeno destinado inicialmente al tejido de la planta y posteriormente al suelo ${ }^{(1)}$; además, entre los cultivos forrajeros, la alfalfa, registra los valores más altos en producción de proteína por unidad de superficie en clima templado ${ }^{(2)}$.

Los principales países productores de alfalfa en el mundo en los últimos cinco años, han sido Estados Unidos de América, Argentina, México, Irán e Italia. Sin embargo, a pesar de que México ocupa el tercer lugar en producción total, registra el mayor rendimiento anual por hectárea en zonas templadas del mundo, con una tasa de crecimiento anual de $0.74 \%{ }^{(3)}$. En el año 2013, en el país, se registraron 31 millones t de forraje verde en 390 mil ha cultivadas, con rendimiento promedio de $80.71 \mathrm{t} \mathrm{ha}^{-1}$ año $^{-1}$; siendo los estados de Chihuahua, Hidalgo y Guanajuato quienes aportaron el $46 \%$ de la producción total del país ${ }^{(4)}$.

La alfalfa es una planta diploide y autotetraploide; de polinización cruzada. Su mejoramiento genético se basa en la selección fenotípica recurrente con pruebas de progenie, para acumular altas frecuencias de alelos deseables en una población ${ }^{(5)}$. En Valles Altos del Centro de México, se han obtenido variedades de alfalfa adaptadas a partir de germoplasma de origen peruano y africano, al considerar como criterios de selección de estas variedades, la persistencia de las plantas, resistencia a plagas y enfermedades, grado de latencia invernal y producción anual ${ }^{(6)}$. Por lo tanto, evaluar la persistencia de los cultivares, la producción de biomasa estacional y los componentes del rendimiento en poblaciones de alfalfa bajo diversas condiciones ambientales, es una herramienta importante para los fitomejoradores en la selección de cultivares superiores para usos y ambientes específicos ${ }^{(7)}$.

Los factores ambientales, el manejo agrícola y los organismos en el ecosistema circundante, interactúan con los cultivos de alfalfa. El dosel vegetal y la estructura de la corona permiten una amplia adaptación. Además, el sistema de raíces profundas y extensas crea un entorno de gran riqueza biológica en el suelo ${ }^{(1)}$. Así, el incremento en rendimiento y sus componentes dependerá de la influencia de los factores ambientales como temperatura, radiación fotosintéticamente activa y fotoperiodo; así como de factores de manejo agrícola como la frecuencia e intensidad de corte o defoliación ${ }^{(8,9)}$.

En Valles Altos del Centro de México, existe una amplia gama de variedades de alfalfa de uso comercial y amplio conocimiento del comportamiento del rendimiento de materia seca en diferentes sistemas de producción y manejo. No obstante, a la fecha no existen trabajos de investigación en los que se hayan generado métodos de selección efectivos para la obtención 
de nuevas variedades más productivas. Al evaluar el comportamiento productivo de cinco variedades comerciales de alfalfa, en Montecillo, Texcoco, Estado de México; se determinó que las variedades comerciales San Miguel y Oaxaca con mayor persistencia, mejor adaptación y mayor rendimiento de materia seca, poseen características deseables para aumentar la producción de forraje ${ }^{(10)}$.

El presente trabajo tuvo como objetivo evaluar en alfalfa, el rendimiento de materia seca y sus componentes, en familias de medios hermanos derivadas de las variedades San Miguel y Oaxaca, y las poblaciones San Miguel x Oaxaca (cruza directa) y Oaxaca x San Miguel (cruza recíproca), en macetas a la intemperie en cortes sucesivos en las diferentes estaciones del año.

\section{Material y métodos}

El experimento se realizó durante los ciclos otoño-invierno-primavera 2013-2014 en el área de invernaderos del Colegio de Postgraduados en Montecillo, Texcoco, Estado de México $\left(19^{\circ} 21^{\prime} \mathrm{N}, 98^{\circ} 55^{\prime} \mathrm{O}\right.$ y altitud de $\left.2,250 \mathrm{msnm}\right)$, clima templado subhúmedo $\left(\mathrm{Cb}(\mathrm{wo})(\mathrm{w})\left(\mathrm{i}^{\prime}\right) \mathrm{g}\right)$, con lluvias en verano, precipitación y temperatura media anual de 636.5 $\mathrm{mm}$ y $15{ }^{\circ} \mathrm{C}$, respectivamente ${ }^{(11)}$. Se utilizaron cuatro poblaciones, conformadas por 100 familias de medios hermanos de las variedades San Miguel y Oaxaca, y las poblaciones San

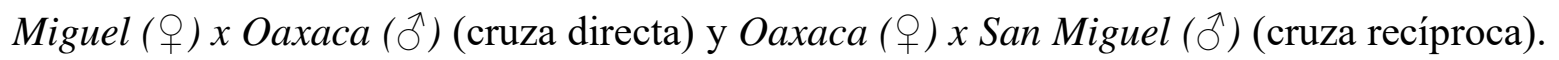
Éstas, obtenidas mediante cruzamiento controlado en campo, entre las variedades San Miguel y Oaxaca, en el ciclo de invierno-primavera 2005 en el Campo Experimental del Colegio de Postgraduados, Montecillo, Texcoco, Estado de México. Las familias de medios hermanos de San Miguel y Oaxaca, y las cruzas directa y recíproca entre éstas, se asignaron bajo un diseño completamente al azar en cada población. La unidad experimental consistió de una familia de medios hermanos o planta individual por maceta dentro de cada población.

La siembra se realizó en cajas para almácigo el 8 de mayo de 2013, al colocar cinco semillas del mismo peso en cada celda. Cuando las plántulas tuvieron una edad de 27 días después de la siembra (dds) se trasplantaron individualmente en macetas de plástico con capacidad de 3 $\mathrm{kg}$. Se utilizó un suelo esterilizado de textura franco-arenosa con densidad aparente de 1.12 $\mathrm{g} \mathrm{cm}^{-3}$, capacidad de campo (CC) de $41.6 \%$ y porcentaje de marchitamiento permanente (PMP) de $28.2 \%$ (Laboratorio Central Universitario, Universidad Autónoma Chapingo, Chapingo, México). Las plántulas en las macetas se colocaron en tarimas de madera a una altura de $1 \mathrm{~m}$ sobre el nivel del piso en un área protegida con malla contra áfidos a la 
intemperie. Se aplicó una dosis de fertilización de 60-140-00 a los 57 dds y una segunda aplicación de la misma dosis, a los $247 \mathrm{dds}$, con urea como fuente de nitrógeno y superfosfato de calcio triple como fuente de fósforo; la alta dosis de fósforo incrementa la actividad de la fosfatasa y el crecimiento del sistema radical ${ }^{(12)}$. Las macetas se regaron cada tercer día, manteniendo la humedad edáfica cercana a capacidad de campo (CC).

Se hizo un corte de uniformización en todas las plantas a los 85 dds y se procedió a realizar cortes sucesivos programados cada cinco semanas en el periodo otoño-invierno 2013-2014, y cada cuatro semanas en el periodo primavera-verano 2014, a una altura de corte de $5 \mathrm{~cm}$ sobre el nivel del suelo. Para cada corte de midieron las siguientes variables:

Altura de planta (AP) en cm, que se determinó con una regla graduada de $1 \mathrm{~m}$ de longitud y $5 \mathrm{~mm}$ de precisión, para lo cual a un lado de cada planta se colocó la regla de forma vertical sobre la superficie del suelo, y un dispositivo de la parte superior de la regla se hizo deslizar hacia abajo hasta tocar las hojas superiores de cada planta, registrándose la altura correspondiente.

Número de tallos (NT) por planta, que se determinó al contar el número total de tallos presentes en cada planta al momento del corte.

Relación hoja:tallo (H:T) por planta; de cada planta se tomaron cuatro tallos al azar, de los cuales se separaron las láminas foliares y los tallos, los que fueron llevados a una estufa por $48 \mathrm{~h}$ a $65^{\circ} \mathrm{C}$, tiempo en que alcanzaron peso constante, el cual se registró con una balanza eléctrica de alta precisión marca Excell modelo BH. Se calculó la relación hoja:tallo al dividir el peso seco del componente hoja (PSH)/peso seco del componente tallo (PST), con la ecuación siguiente H:T=PSH/PST; peso seco (PS) por planta (g); después de separar los cuatro tallos para la relación H:T, el resto de cada planta se colocó en bolsas de papel, las cuales se llevaron a una estufa por $48 \mathrm{~h} \mathrm{a} 65^{\circ} \mathrm{C}$, tiempo en que alcanzaron peso constante.

Rendimiento de materia seca (RMS) por planta (g); se calculó al sumar el peso seco por planta más el peso seco de los tallos y láminas foliares utilizados para calcular la relación hoja:tallo.

Tasa absoluta de crecimiento (TAC) por planta $\left(\mathrm{g} \mathrm{MS} \mathrm{d}^{-1}\right)$; se calculó al dividir el rendimiento de materia seca por planta/el número de días $(\mathrm{t})$ transcurrido entre un corte y el siguiente $(\mathrm{TAC}=\mathrm{RMS} / \mathrm{t})$.

Eficiencia en el uso de la radiación (EUR) por planta $\left(\mathrm{g} \mathrm{MS} \mathrm{MJ}^{-1}\right)$; se determinó al dividir el RMS entre la radiación solar acumulada diariamente (datos obtenidos de la estación meteorológica de la Universidad Autónoma Chapingo) en el periodo entre un corte y el siguiente, durante el experimento.

La temperatura máxima y mínima del aire se obtuvo con un termómetro de máxima y mínima, marca Taylor modelo 5458P, colocado junto las plantas a una altura de $2 \mathrm{~m}$ sobre el 
nivel del piso. La cantidad de precipitación se determinó con un pluviómetro de acumulación semanal, colocado junto a las plantas durante el experimento. La temperatura máxima varió de 20.4 a $29.9^{\circ} \mathrm{C}$, la mínima de 1.0 a $11.4^{\circ} \mathrm{C}$ y se registró una precipitación total de $583 \mathrm{~mm}$, durante la estación de crecimiento y cortes subsecuentes (Figura 1). Las temperaturas registradas indicaron que las plantas estuvieron sujetas a condiciones térmicas favorables para su crecimiento, al considerar que la temperatura base $(\mathrm{Tb})$ en clima templado es de $1{ }^{\circ} \mathrm{C}$ cuando la temperatura del aire (Ta) es $\leq 15^{\circ} \mathrm{C}$ y Tb de $5^{\circ} \mathrm{C}$ cuando $\mathrm{Ta} \geq 15^{\circ} \mathrm{C}^{(8)}$.

Figura 1: Temperatura máxima, media y mínima promedio, y precipitación acumulada durante el experimento en Montecillo ciclo 2013-2014

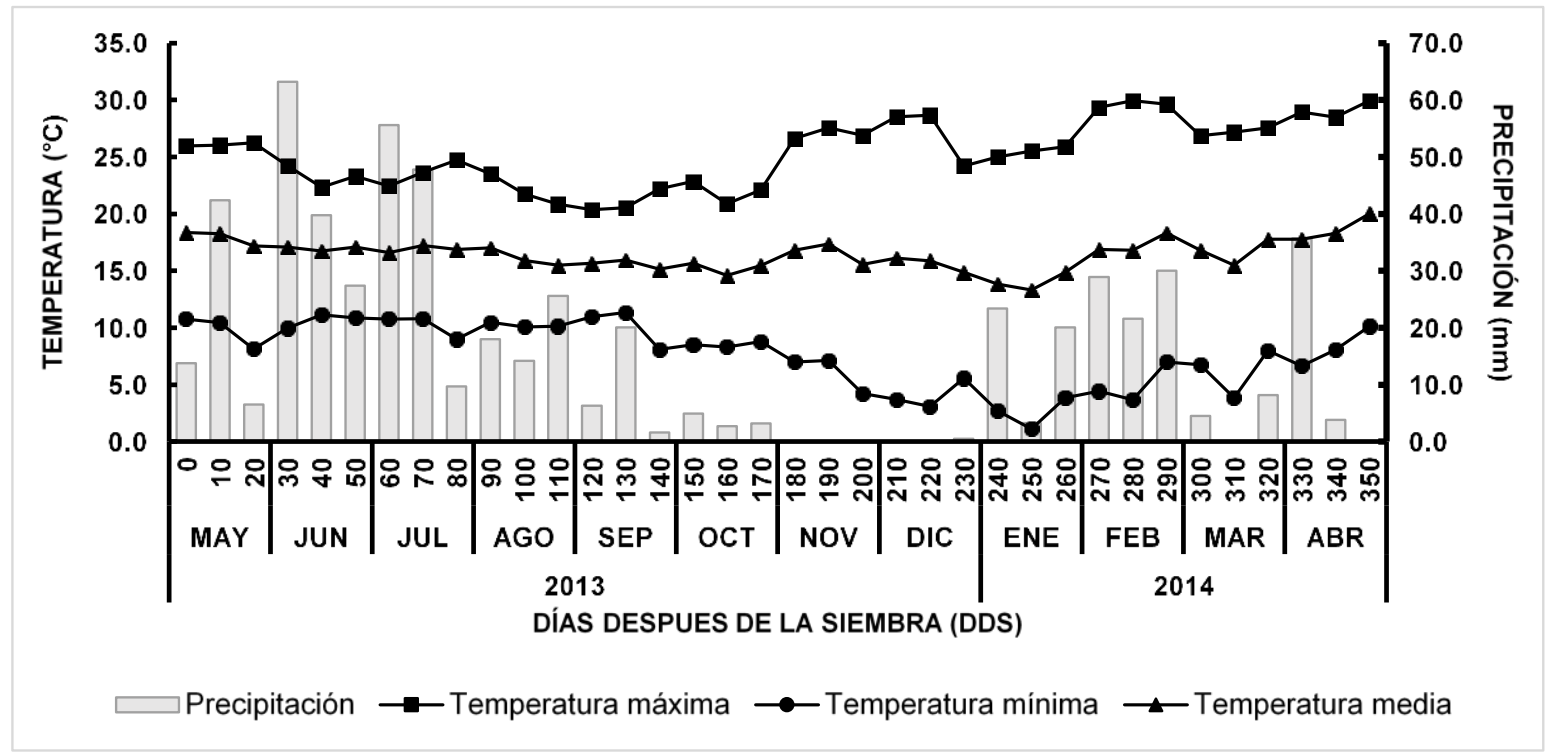

El análisis estadístico utilizado para determinar el efecto conjunto de estaciones del año, cortes, poblaciones y familias se hizo con el programa SAS para Windows, versión 9.1, de acuerdo al siguiente modelo:

$$
\begin{gathered}
\mathrm{Y}_{i j k}=\mu+\mathrm{Pob}_{i}+\operatorname{Fam}(\text { Pob })_{i k}+\text { Est }_{j}+\text { Corte }(E s t)_{j l}+\left(\text { Pob*Est }_{i j}+\text { Corte }_{\left(\text {Pob }^{*} E s t\right.}\right)_{i j l}+ \\
\text { Fam }\left(\text { Pob }^{*} \text { Est }\right)_{i j k}+\mathrm{E}_{i j k} ;
\end{gathered}
$$

Donde:

$\mathbf{Y}_{i j k}$ : representa el valor de la variable de respuesta en la Población $i$ del nivel $j$ de Estación, nivel $k$ de Familia y nivel $l$ de Corte;

$\boldsymbol{\mu}$ : es la media general;

Pob $_{i}$ : es el efecto de la Población al nivel $i=1,2,3$ y 4;

Fam(Pob $)_{i k}$ : es el efecto de Familias anidadas a las Poblaciones al nivel $i$ y $k$; 
Est $_{j}$ : es el efecto de la estación del año al nivel $j=1,2$ y 3 ;

Corte $(\mathbf{E s t})_{j l}$ : es el efecto de los cortes anidados a las estaciones del año al nivel $j$ y $l$;

$\left(\text { Pob }^{*} \text { Est }\right)_{i j}$ : es el efecto de la interacción Población x Estación del año al nivel $i$ y $j$;

Corte $(\mathbf{P o b} * \mathbf{E s t})_{i j l}$ : es el efecto de la interacción Población x Estación anidadas a los Cortes al nivel $i, j$ y $l$;

Fam $\left(\text { Pob }^{*} \text { Est }\right)_{i j k}$ : es el efecto de la interacción Población x Estación anidadas a las Familias al nivel $i, j, k$;

$\mathbf{E}_{i j k}$ : es el error experimental.

La comparación de medias se hizo con la diferencia mínima significativa (DMS, $P \leq 0.05$ ), con el programa estadístico SAS para Windows.

\section{Resultados y discusión}

La variabilidad en rendimiento de materia seca (RMS), tasa absoluta de crecimiento (TAC), eficiencia en el uso de radiación (EUR), relación hoja:tallo (H:T), altura (AP) y número de tallos (NT) por planta fue altamente significativa $(P \leq 0.01)$ entre poblaciones, familias de medios hermanos dentro de cada población, estaciones del año, cortes dentro de estaciones del año, poblaciones $\mathrm{x}$ estaciones del año, familias de medios hermanos dentro de poblaciones $\mathrm{x}$ estaciones del año y cortes dentro de poblaciones $\mathrm{x}$ estaciones del año, excepto, en altura de planta para poblaciones $(P \leq 0.05)$.

El análisis de varianza de acuerdo al modelo utilizado para determinar los efectos de población, estación del año, cortes y familias de medios hermanos mostró que el ambiente y el genotipo influyeron en el rendimiento de forraje y sus componentes. La variación entre estaciones del año y cortes fue de mayor magnitud que la variación observada entre poblaciones, familias dentro de poblaciones $\mathrm{y}$ poblaciones $\mathrm{x}$ cortes, poblaciones $\mathrm{x}$ estaciones, familias dentro poblaciones $\mathrm{x}$ estaciones del año $\mathrm{y}$ cortes dentro de poblaciones $\mathrm{x}$ estaciones (Cuadro 1). El rendimiento de variedades de alfalfa es dependiente del efecto del genotipo, el ambiente y la interacción genotipo $\mathrm{x}$ ambiente. Se ha demostrado que las variedades antiguas de alfalfa, liberadas en Estados Unidos en los últimos 60 años, no difirieron en rendimiento de forraje con respecto a las variedades de reciente liberación, cuando se compararon con densidades de población similares. Sin embargo, cuando los factores 
bióticos y abióticos afectaron la densidad de población, se pudieron observar ganancias en rendimiento en las variedades modernas hasta del $10 \%$ por década $^{(13)}$.

Cuadro 1: Análisis de varianza en el rendimiento de materia seca (RMS), tasa absoluta de crecimiento (TAC), relación hoja:tallo (H:T), número de tallos (NT) y altura de planta (AP), en siete cortes y cuatro poblaciones de alfalfa

\begin{tabular}{lcccccc}
\hline \multirow{2}{*}{ Fuente de variación } & Grados de & \multicolumn{5}{c}{ Cuadrados medios } \\
\cline { 3 - 7 } & libertad & RMS & TC & H:T & Altura & Tallos (n) \\
\hline Poblaciones & 3 & $94.1^{* *}$ & $0.092^{* *}$ & $3.21^{* *}$ & $136.93^{*}$ & $726.58^{* *}$ \\
Familias (Pobl.) & 375 & $18.9^{* *}$ & $0.019^{* *}$ & $0.17^{* *}$ & $142.33^{* *}$ & $112.42^{* *}$ \\
Estaciones & 2 & $5581.8^{* *}$ & $8.816^{* *}$ & $21.94^{* *}$ & $37427.93^{* *}$ & $16679.87^{* *}$ \\
Cortes (Estac.) & 4 & $900.5^{* *}$ & $0.735^{* *}$ & $20.32^{* *}$ & $24132.03^{* *}$ & $2166.01^{* *}$ \\
Poblaciones*Estaciones & 6 & $13.7^{* *}$ & $0.013^{* *}$ & $0.40^{* *}$ & $231.08^{* *}$ & $322.21^{* *}$ \\
Familias (Pobl..*Estac.) & 12 & $11.1^{* *}$ & $0.009^{* *}$ & $0.64^{* *}$ & $188.72^{* *}$ & $48.02^{* *}$ \\
Cortes (Pobl.*Estac.) & 741 & $5.0^{* *}$ & $0.005^{* *}$ & $0.07^{* *}$ & $52.75^{* *}$ & $18.68^{* *}$ \\
Error & 2586 & 2.4 & 0.002 & 0.59 & 26.74 & 7.38 \\
Media & & 6.6 & 0.198 & 1.22 & 49.98 & 13.54 \\
CV, \% & & 23.6 & 22.293 & 19.86 & 10.34 & 20.06 \\
\hline
\end{tabular}

$\mathrm{CV}=$ coeficiente de variación; * $(P \leq 0.05) ;{ }^{* *}(P \leq 0.001)$.

En RMS se detectaron diferencias altamente significativas $(P \leq 0.01)$ entre estaciones del año; en primavera fue $57 \%$ mayor que en otoño y $33 \%$ mayor que en invierno en promedio de las cuatro poblaciones (Cuadro 2). Este incremento en el RMS se puede atribuir a las altas temperaturas registradas en primavera (máxima $=30^{\circ} \mathrm{C}$ y mínima $=8{ }^{\circ} \mathrm{C}$ ) en comparación con invierno (máxima $=27{ }^{\circ} \mathrm{C}$ y mínima $=4{ }^{\circ} \mathrm{C}$ ) y otoño (máxima $=25^{\circ} \mathrm{C}$ y mínima $=7{ }^{\circ} \mathrm{C}$ ), que promovieron una mayor tasa de rebrote de yemas de las coronas formadas en otoño del año anterior ${ }^{(14)}$. El conocimiento del efecto de la temperatura estacional en la dinámica de crecimiento de la alfalfa, permite determinar la frecuencia e intensidad de corte, para optimizar la producción y persistencia de los cultivares ${ }^{(9,15)}$. También, se detectaron diferencias significativas $(P \leq 0.05)$ entre poblaciones; las poblaciones derivadas de las cruzas San Miguel x Oaxaca y Oaxaca x San Miguel, produjeron 11 y $10 \%$ mayor rendimiento de materia seca que el promedio de las variedades originales en todas las estaciones del año. En un estudio realizado en Serbia, se observó que el incremento en la producción de forraje de alfalfa con relación a los progenitores, fue de $9.1 \%$ en la progenie $F_{1}$ (cruzamientos dialélicos) y $8.1 \%$ en la progenie $\mathrm{OP}_{1}$ (polinización abierta); así el cruzamiento de hermanos completos o medios hermanos, conlleva un incremento significativo en el rendimiento de materia seca y sus componentes ${ }^{(16)}$. 
Cuadro 2: Rendimiento de materia seca (RMS), tasa absoluta de crecimiento (TAC), eficiencia en el uso de la radiación (EUR); relación hoja:tallo (H:T), altura de planta (AP) y número de tallos (NT), promedio por estación y población de alfalfa

\begin{tabular}{|c|c|c|c|c|c|c|c|c|}
\hline Estación & Variables & $\begin{array}{c}\text { San } \\
\text { Miguel }\end{array}$ & Oaxaca & $\begin{array}{c}\text { San Miguel } \\
\text { x Oaxaca }\end{array}$ & $\begin{array}{l}\text { Oaxaca x } \\
\text { San Miguel }\end{array}$ & Promedio & DMS & Sign. \\
\hline \multirow[t]{6}{*}{ Otoño } & RMS & $4.02 \mathrm{Cc}$ & $4.35 \mathrm{Cbc}$ & $4.60 \mathrm{Cab}$ & $4.93 \mathrm{Ca}$ & $4.48 \mathrm{C}$ & 0.42 & * \\
\hline & TAC & $0.11 \mathrm{Cc}$ & $0.12 \mathrm{Cbc}$ & $0.13 \mathrm{Cab}$ & $0.14 \mathrm{Ca}$ & $0.128 \mathrm{C}$ & 0.01 & * \\
\hline & EUR & 0.45 & 0.49 & 0.48 & 0.53 & $0.50 \mathrm{C}$ & & \\
\hline & $\mathrm{H}: \mathrm{T}$ & 1.30 Aab & $1.21 \mathrm{Ab}$ & $1.38 \mathrm{Aa}$ & 1.36 Аа & $1.31 \mathrm{~A}$ & 0.09 & * \\
\hline & $\mathrm{AP}$ & $48.8 \mathrm{~B}$ & $47.4 \mathrm{~B}$ & $47.1 \mathrm{~B}$ & $48.4 \mathrm{~B}$ & $48.0 \mathrm{~B}$ & 1.97 & NS \\
\hline & NT & $10 \mathrm{C}$ & $9 C$ & $10 \mathrm{C}$ & $10 \mathrm{C}$ & $10 \mathrm{C}$ & 1.07 & NS \\
\hline \multirow[t]{6}{*}{ Invierno } & RMS & $7.03 \mathrm{Bab}$ & $6.52 \mathrm{Bb}$ & $7.5 \mathrm{Ba}$ & $7.3 \mathrm{Ba}$ & $7.1 \mathrm{~B}$ & 0.68 & * \\
\hline & TAC & $0.20 \mathrm{Bab}$ & $0.19 \mathrm{Bb}$ & $0.21 \mathrm{Ba}$ & $0.21 \mathrm{Ba}$ & $0.20 \mathrm{~B}$ & 0.02 & * \\
\hline & EUR & 0.75 & 0.70 & 0.80 & 0.78 & $0.75 \mathrm{~B}$ & & \\
\hline & $\mathrm{H}: \mathrm{T}$ & $1.11 \mathrm{Bc}$ & $1.22 \mathrm{Ab}$ & 1.32 Ba & $1.31 \mathrm{Ba}$ & $1.24 \mathrm{~B}$ & 0.05 & * \\
\hline & $\mathrm{AP}$ & $47.0 \mathrm{C}$ & $46.7 \mathrm{~B}$ & $46.8 \mathrm{~B}$ & $48.0 \mathrm{~B}$ & $47.1 \mathrm{~B}$ & 1.62 & NS \\
\hline & NT & $17 \mathrm{Ba}$ & $14 \mathrm{Bb}$ & $14 \mathrm{Bb}$ & $15 \mathrm{Bb}$ & $15 \mathrm{~B}$ & 1.41 & * \\
\hline \multirow[t]{6}{*}{ Primavera } & RMS & $10.10 \mathrm{Ac}$ & 10.14 Abc & $11.25 \mathrm{Aa}$ & $10.93 \mathrm{Aab}$ & $10.60 \mathrm{~A}$ & 0.82 & * \\
\hline & TAC & $0.36 \mathrm{Ac}$ & $0.36 \mathrm{Abc}$ & $0.40 \mathrm{Aa}$ & $0.39 \mathrm{Aab}$ & $0.38 \mathrm{~A}$ & 0.03 & * \\
\hline & EUR & 0.90 & 0.91 & 1.00 & 0.98 & $0.95 \mathrm{~A}$ & & \\
\hline & $\mathrm{H}: \mathrm{T}$ & $0.87 \mathrm{Cb}$ & $0.85 \mathrm{Bb}$ & $0.98 \mathrm{Ca}$ & $0.96 \mathrm{Ca}$ & $0.91 \mathrm{C}$ & 0.06 & * \\
\hline & $\mathrm{AP}$ & $60.3 \mathrm{Ab}$ & $64.4 \mathrm{Aa}$ & $63.1 \mathrm{Aa}$ & $63.7 \mathrm{Aa}$ & $62.9 \mathrm{~A}$ & 2.24 & * \\
\hline & NT & $23 \mathrm{Aa}$ & $17 \mathrm{Ac}$ & $20 \mathrm{Ab}$ & $17 \mathrm{Ac}$ & $19 \mathrm{~A}$ & 2.02 & $* *$ \\
\hline \multirow[t]{6}{*}{ Promedio } & RMS & $7.0 \mathrm{~b}$ & $7.0 \mathrm{~b}$ & $7.8 \mathrm{a}$ & $7.7 \mathrm{a}$ & & & \\
\hline & TAC & $0.23 b$ & $0.22 b$ & $0.25 a$ & $0.25 a$ & & & \\
\hline & EUR & 0.64 & 0.64 & 0.70 & 0.70 & & & \\
\hline & $\mathrm{H}: \mathrm{T}$ & $1.09 b$ & $1.09 b$ & $1.23 a$ & $1.21 \mathrm{a}$ & & & \\
\hline & AP & 52.0 & 52.9 & 52.4 & 53.4 & & & \\
\hline & NT & 17 a & $13 b$ & $15 a b$ & $14 \mathrm{~b}$ & & & \\
\hline \multirow[t]{6}{*}{ DMS } & RMS & 0.42 & 0.45 & 0.49 & 0.43 & & & \\
\hline & TAC & 0.01 & 0.01 & 0.02 & 0.01 & & & \\
\hline & EUR & & & & & & & \\
\hline & $\mathrm{H}: \mathrm{T}$ & 0.05 & 0.06 & 0.06 & 0.04 & & & \\
\hline & AP & 1.58 & 1.53 & 1.49 & 1.53 & & & \\
\hline & $\mathrm{NT}$ & 1.16 & 0.88 & 0.96 & 0.85 & & & \\
\hline \multirow[t]{6}{*}{ Sign. } & RMS & $* *$ & $* *$ & $* *$ & $* *$ & & & \\
\hline & $\mathrm{TAC}$ & $* *$ & $* *$ & $* *$ & $* *$ & & & \\
\hline & EUR & & & & & & & \\
\hline & $\mathrm{H}: \mathrm{T}$ & $* *$ & ** & $* *$ & ** & & & \\
\hline & $\mathrm{AP}$ & $* *$ & ** & $* *$ & $* *$ & & & \\
\hline & NT & $* *$ & $* *$ & $* *$ & $* *$ & & & \\
\hline
\end{tabular}

Diferente literal en mayúsculas en cada columna indica diferencias significativas entre estaciones $(P \leq 0.01)$; diferente literal en minúsculas en cada hilera indica diferencias significativas entre poblaciones $(P \leq 0.05)$;

DMS=Diferencia mínima significativa; ${ }^{*}(P \leq 0.05){ }^{* *}(P \leq 0.01)$, NS $=$ no significativas. 
En RMS por cortes dentro de estaciones del año se detectaron diferencias altamente significativas $(P \leq 0.01)$ (Cuadro 3); el RMS promedio por corte fue mayor en los cortes de marzo y abril, que el promedio de todos los cortes (6.52 $\left.\mathrm{g} \mathrm{MS} \mathrm{planta}^{-1}\right)$. Esta mayor acumulación de forraje en marzo y abril, se debió a la presencia de temperaturas más altas (máxima $=30{ }^{\circ} \mathrm{C}$ y mínima $=8{ }^{\circ} \mathrm{C}$ ), fotoperiodo más largo $(12.4 \mathrm{~h})$ y radiación fotosintéticamente activa más alta $\left(10 \mathrm{MJ} \mathrm{m}^{-2} \mathrm{~d}^{-1}\right)$ que en septiembre, octubre y noviembre (máxima $=25^{\circ} \mathrm{C}$, mínima $=7^{\circ} \mathrm{C}$, fotoperiodo $=11.6 \mathrm{~h}$ y radiación fotosintéticamente activa $=$ $8 \mathrm{MJ} \mathrm{m}^{-2} \mathrm{~d}^{-1}$ ), y diciembre, enero y febrero (máxima $=27{ }^{\circ} \mathrm{C}$, mínima $=4{ }^{\circ} \mathrm{C}$, fotoperiodo $=$ $11.2 \mathrm{~h}$ y radiación fotosintéticamente activa $\left.=8 \mathrm{MJ} \mathrm{m}^{-2} \mathrm{~d}^{-1}\right)$. En un estudio donde se evaluó el efecto de la temperatura sobre el rendimiento, al utilizar un análisis de regresión múltiple con datos climatológicos y de rendimiento colectados a través de 21 años, en Minnesota, Estados Unidos, en donde se determinó que el rendimiento de alfalfa en primavera para el segundo año de establecimiento, fue afectado positivamente por las temperaturas máximas de otoño y primavera, y temperaturas mínimas en invierno; la temperatura determinó el $50 \%$ de la variabilidad en el rendimiento ${ }^{(17)}$. De la misma manera, otros autores ${ }^{(18)}$ determinaron que el mejor crecimiento de alfalfa ocurrió a temperaturas diurnas de 15 a $25{ }^{\circ} \mathrm{C}$ y nocturnas de 10 a $20^{\circ} \mathrm{C}$, rango óptimo para una alta tasa fotosintética y mayor absorción de $\mathrm{CO}_{2}$ por la planta.

Cuadro 3: Rendimiento de materia seca (RMS) promedio por corte en las diferentes poblaciones de alfalfa

\begin{tabular}{|c|c|c|c|c|c|c|c|}
\hline \multirow[b]{2}{*}{ Cortes } & \multicolumn{4}{|c|}{ Poblaciones } & \multirow[b]{2}{*}{ Promedio } & \multirow[b]{2}{*}{ Sign. } & \multirow[b]{2}{*}{ DMS } \\
\hline & San Miguel & Oaxaca & $\begin{array}{c}\text { San Miguel x } \\
\text { Oaxaca }\end{array}$ & $\begin{array}{c}\text { Oaxaca x } \\
\text { San Miguel }\end{array}$ & & & \\
\hline 30/Sep/13 & $4.70 \mathrm{~Eb}$ & $4.49 \mathrm{~Eb}$ & $5.33 \mathrm{Da}$ & 5.30 EDa & $4.97 \mathrm{E}$ & * & 0.59 \\
\hline $29 / 0 \mathrm{ct} / 13$ & $3.60 \mathrm{~Gb}$ & $4.37 \mathrm{Ea}$ & $3.46 \mathrm{~Eb}$ & $4.27 \mathrm{Fa}$ & $3.92 \mathrm{~F}$ & * & 0.55 \\
\hline $28 /$ Nov/13 & 4.16 Fb & $4.48 \mathrm{~Eb}$ & $5.16 \mathrm{Da}$ & $5.21 \mathrm{Ea}$ & $4.76 \mathrm{E}$ & * & 0.60 \\
\hline 06/Ene/14 & $5.33 \mathrm{Db}$ & $5.10 \mathrm{Db}$ & $6.03 \mathrm{Ca}$ & 5.74 Dab & $5.56 \mathrm{D}$ & * & 0.66 \\
\hline 13/Feb/14 & $6.48 \mathrm{Ca}$ & $5.66 \mathrm{Cb}$ & 6.27 Cab & $6.56 \mathrm{Ca}$ & $6.25 \mathrm{C}$ & * & 0.66 \\
\hline 20/Mar/14 & $9.37 \mathrm{Bb}$ & $8.95 \mathrm{Bb}$ & 10.33 Ba & $9.56 \mathrm{Bab}$ & $9.56 \mathrm{~B}$ & * & 0.89 \\
\hline 17/Abr/14 & $10.10 \mathrm{Ac}$ & 10.14 Abc & $11.25 \mathrm{Aa}$ & 10.93 Aab & $10.61 \mathrm{~A}$ & * & 0.82 \\
\hline Promedio & $6.30 \mathrm{~b}$ & $6.21 \mathrm{~b}$ & $6.86 \mathrm{a}$ & $6.81 \mathrm{a}$ & & & \\
\hline Sign. & $* *$ & $* *$ & $* *$ & ** & & & \\
\hline DMS & 0.51 & 0.52 & 0.55 & 0.51 & & & \\
\hline
\end{tabular}

Diferente literal en mayúsculas en cada columna indica diferencias significativas entre cortes $(P \leq 0.01)$; diferente literal en minúsculas en cada hilera indica diferencias significativas entre poblaciones $(P \leq 0.05)$; DMS=Diferencia mínima significativa; * $(P \leq 0.05) ;{ }^{* *}(P \leq 0.01)$.

Se detectaron también diferencias significativas $(P \leq 0.05)$ en RMS entre poblaciones en cada corte realizado (Cuadro 3); la población San Miguel x Oaxaca produjo mayor RMS que el promedio de las poblaciones originales en $14,16,14,11$ y $10 \%$, para los cortes de 
septiembre, noviembre, enero, marzo y abril, respectivamente. La población Oaxaca $x$ San Miguel produjo 13 \% más RMS en septiembre y 17 \% más RMS en octubre y noviembre. En todos los cortes realizados las poblaciones originales de Oaxaca y San Miguel presentaron menor RMS que las poblaciones de familias de medios hermanos, excepto, la población Oaxaca en el corte de octubre y la población San Miguel en el corte de febrero, meses en que presentaron rendimientos estadísticamente iguales a la población Oaxaca x San Miguel. La variabilidad observada entre las poblaciones originales y las poblaciones de las cruzas en cada corte realizado, muestra posibilidades de seleccionar familias superiores en rendimiento de materia seca, con ganancias genéticas del orden de $10 \%$ por ciclo de selección ${ }^{(19)}$. Así, la evaluación de progenitores con pruebas de progenie, mediante polinización abierta es eficaz para evaluar el rendimiento de materia seca y componentes de calidad; la prueba es eficaz si se realizan cruzas entre poblaciones genéticamente diversas, con el fin de producir la combinación de híbridos que pueden ser usados como donadores de genes en el desarrollo de nuevos cultivares sintéticos ${ }^{(20)}$.

La tasa de crecimiento del cultivo es el producto de la incidencia de luz sobre el dosel vegetal, la cantidad de luz interceptada por el área foliar y la eficiencia en el uso de la energía para la producción de materia seca ${ }^{(21)}$. En la TAC se detectaron diferencias altamente significativas $(P \leq 0.01)$ entre estaciones del año, la TAC promedio por estación más alta fue $0.38 \mathrm{~g} \mathrm{~d}^{-1}$ en primavera y la más baja fue $0.13 \mathrm{~g} \mathrm{~d}^{-1}$ en otoño (Cuadro 2). Estos resultados concuerdan con lo observado en Valles Centrales de Oaxaca, donde las mayores TAC se registraron durante la primavera, seguido de invierno, verano y otoño ${ }^{(22)}$; en cambio, en Montecillo, Texcoco, Estado de México, se observó que la TAC promedio en 11 variedades de alfalfa fue mayor en verano, primavera e invierno que en otoño ${ }^{(23)}$. Las altas TAC observadas en primavera e invierno, además de ser influenciadas por las condiciones ambientales, también tienen influencia de la acumulación de carbono $(\mathrm{C})$ y nitrógeno $(\mathrm{N})$ en la raíz y la corona. Las reservas de carbohidratos acumulados en dichos órganos, determinan la TAC del rebrote, esto principalmente después del corte y durante el rebrote temprano en primavera ${ }^{(24)}$.

Al comparar los valores de la TAC entre poblaciones en las estaciones del año, se observaron diferencias significativas $P \leq 0.05$ ), siendo San Miguel $x$ Oaxaca y Oaxaca $x$ San Miguel, las que registraron valores de mayor magnitud con relación a las poblaciones originales (Cuadro 2). Se ha demostrado que en poblaciones obtenidas mediante cruzamiento, se incrementa la acumulación de alelos deseables, es decir, la acción génica aditiva, que conlleva a un aumento significativo en la producción ${ }^{(5)}$.

En la TAC por corte se detectaron diferencias significativas $(P \leq 0.05)$ entre poblaciones (Cuadro 4); San Miguel x Oaxaca y Oaxaca x San Miguel registraron una TAC mayor en $0.010 \mathrm{~g}$ de $\mathrm{MS}_{\mathrm{S}}$ planta $^{-1}$ y $0.008 \mathrm{~g}$, que el promedio de las cuatro poblaciones originales $(0.198$ g). Tambien, la TAC, presentó diferencias altamente significativas $(P \leq 0.01)$ para los siete cortes; el corte de abril fue mayor en $0.182 \mathrm{~g}$, respectivamente, comparada con la media de todos los cortes $(0.197 \mathrm{~g})$. Esta variación es efecto de las condiciones ambientales favorables, 
temperaturas medias elevadas, fotoperiodo largo y mayor radiación fotosinteticamente activa en primavera que en las otras estaciones del año.

Cuadro 4: Tasa absoluta de crecimiento promedio por corte en las diferentes poblaciones de alfalfa

\begin{tabular}{|c|c|c|c|c|c|c|c|}
\hline \multirow[b]{2}{*}{ Cortes } & \multicolumn{4}{|c|}{ Poblaciones } & \multirow[b]{2}{*}{ Promedio } & \multirow[b]{2}{*}{ Sign. } & \multirow[b]{2}{*}{ DMS } \\
\hline & $\begin{array}{c}\text { San } \\
\text { Miguel }\end{array}$ & Oaxaca & $\begin{array}{c}\text { San Miguel } \\
\text { x Oaxaca }\end{array}$ & $\begin{array}{c}\text { Oaxaca x } \\
\text { San Miguel }\end{array}$ & & & \\
\hline 03/Sep/13 & $0.134 \mathrm{~Eb}$ & $0.128 \mathrm{~Eb}$ & $0.152 \mathrm{Da}$ & $0.151 \mathrm{Da}$ & $0.142 \mathrm{E}$ & * & 0.017 \\
\hline $29 / 0 c t / 13$ & $0.102 \mathrm{Fb}$ & $0.123 \mathrm{Ea}$ & $0.099 \mathrm{~Eb}$ & $0.122 \mathrm{Ea}$ & $0.111 \mathrm{~F}$ & * & 0.021 \\
\hline 28/Nov/13 & $0.119 \mathrm{~Eb}$ & $0.128 \mathrm{~Eb}$ & $0.147 \mathrm{Da}$ & $0.149 \mathrm{Da}$ & $0.136 \mathrm{E}$ & * & 0.017 \\
\hline 06/Ene/14 & $0.152 \mathrm{Db}$ & $0.146 \mathrm{Db}$ & $0.172 \mathrm{Ca}$ & $0.164 \mathrm{Dab}$ & $0.159 \mathrm{D}$ & * & 0.025 \\
\hline 13/Feb/14 & $0.185 \mathrm{Ca}$ & $0.162 \mathrm{Cb}$ & 0.179 Cab & $0.187 \mathrm{Ca}$ & $0.179 \mathrm{C}$ & * & 0.019 \\
\hline 20/Mar/14 & $0.268 \mathrm{Bb}$ & $0.256 \mathrm{Bb}$ & $0.295 \mathrm{Ba}$ & $0.273 \mathrm{Bab}$ & $0.273 \mathrm{~B}$ & * & 0.025 \\
\hline 17/Abr/14 & $0.361 \mathrm{Ac}$ & $0.362 \mathrm{Abc}$ & $0.402 \mathrm{Aa}$ & $0.390 \mathrm{Aab}$ & $0.379 \mathrm{~A}$ & * & 0.029 \\
\hline Promedio & $0.191 b$ & $0.188 \mathrm{~b}$ & $0.208 a$ & $0.206 \mathrm{a}$ & & & \\
\hline Sign. & $* *$ & $* *$ & $* *$ & ** & ** & & \\
\hline DMS & 0.015 & 0.016 & 0.017 & 0.016 & & & \\
\hline
\end{tabular}

Diferente literal en mayúsculas en cada columna indica diferencias significativas entre cortes ( $P \leq 0.01)$; diferente literal en minúsculas en cada hilera indica diferencias significativas entre poblaciones $(P \leq 0.05)$; DMS=Diferencia mínima significativa; ${ }^{*}(P \leq 0.05) ;{ }^{* *}(P \leq 0.01)$.

La eficiencia con que las plantas capturan la radiación solar y la convierten en biomasa, está determinada por la eficiencia en el uso de la radiación (EUR), la cual fue mayor en primavera (0.95 $\left.\mathrm{g} \mathrm{MS} \mathrm{MJ}^{-1}\right)$ que en invierno (0.75 g) y otoño (0.50 g) (Cuadro 2). La EUR también mostró diferencias altamente significativas $(P \leq 0.01)$ entre cortes, siendo mayor para el corte de marzo (0.96) y abril (0.95), y menor en el mes de octubre (0.44). Las familias de medios hermanos derivadas de las poblaciones San Miguel x Oaxaca y Oaxaca $x$ San Miguel mostraron $10 \%$ mayor EUR que las poblaciones San Miguel y Oaxaca; las poblaciones San Miguel x Oaxaca y Oaxaca x San Miguel mostraron mayor EUR en todos los cortes, excepto la población San Miguel en el corte de febrero y la población Oaxaca en Octubre (Cuadro 5). Otros estudios han determinado que la EUR en el cultivo de la alfalfa, puede incrementar el rendimiento en $0.6 \mathrm{~g}$ de $\mathrm{MS} \mathrm{MJ}{ }^{-1}$ a una temperatura de $6^{\circ} \mathrm{C}$ y $1.6 \mathrm{~g}$ a una temperatura de 18 ${ }^{\circ} \mathrm{C}^{(25)}$. 
Revista Mexicana de Ciencias Pecuarias Volumen 9 Número 32018

Cuadro 5: Eficiencia en el uso de la radiación (EUR) promedio por corte de las diferentes poblaciones de alfalfa

\begin{tabular}{|c|c|c|c|c|c|c|c|}
\hline \multirow[b]{2}{*}{ Cortes } & \multicolumn{4}{|c|}{ Poblaciones } & \multirow[b]{2}{*}{ Promedio } & \multirow[b]{2}{*}{ Sign. } & \multirow[b]{2}{*}{ DMS } \\
\hline & San Miguel & Oaxaca & $\begin{array}{c}\text { San Miguel } \\
\text { x Oaxaca }\end{array}$ & $\begin{array}{c}\text { Oaxaca } x \\
\text { San Miguel }\end{array}$ & & & \\
\hline 03/Sep/13 & $0.47 \mathrm{~Eb}$ & $0.45 \mathrm{~Eb}$ & $0.53 \mathrm{Da}$ & $0.53 \mathrm{EDa}$ & $0.50 \mathrm{D}$ & * & 0.14 \\
\hline $29 / 0 \mathrm{ct} / 13$ & $0.40 \mathrm{~Gb}$ & $0.49 \mathrm{Ea}$ & $0.38 \mathrm{~Eb}$ & $0.47 \mathrm{Fa}$ & $0.44 \mathrm{~F}$ & * & 0.08 \\
\hline $28 /$ Nov/13 & $0.48 \mathrm{Fb}$ & $0.51 \mathrm{~Eb}$ & $0.59 \mathrm{Da}$ & $0.60 \mathrm{Ea}$ & $0.55 \mathrm{E}$ & * & 0.11 \\
\hline 06/Ene/14 & $0.60 \mathrm{Db}$ & $0.57 \mathrm{Db}$ & $0.68 \mathrm{Ba}$ & $0.64 \mathrm{Dab}$ & $0.62 \mathrm{C}$ & * & 0.07 \\
\hline 13/Feb/14 & $0.72 \mathrm{Ca}$ & $0.63 \mathrm{Cb}$ & $0.70 \mathrm{Bab}$ & $0.73 \mathrm{Ca}$ & $0.69 \mathrm{~B}$ & * & 0.05 \\
\hline 20/Mar/14 & $0.94 \mathrm{Bb}$ & $0.90 \mathrm{Bb}$ & $1.03 \mathrm{Aa}$ & $0.96 \mathrm{Bab}$ & $0.96 \mathrm{~A}$ & * & 0.05 \\
\hline 17/Abr/14 & $0.90 \mathrm{Ac}$ & $0.91 \mathrm{Abc}$ & $1.00 \mathrm{Aa}$ & $0.98 \mathrm{Aab}$ & $0.95 \mathrm{~A}$ & * & 0.06 \\
\hline Promedio & $0.64 \mathrm{~b}$ & $0.64 \mathrm{~b}$ & $0.70 \mathrm{a}$ & $0.70 \mathrm{a}$ & & & \\
\hline Sign. & $* *$ & $* *$ & ** & ** & & & \\
\hline DMS & 0.12 & 0.11 & 0.13 & 0.12 & & & \\
\hline
\end{tabular}

Diferente literal en mayúsculas en cada columna indica diferencias significativas entre cortes $(P \leq 0.01)$; diferente literal en minúsculas en cada hilera indica diferencias significativas entre poblaciones $(P \leq 0.05)$; DMS=Diferencia mínima significativa; ${ }^{*}(P \leq 0.05){ }^{* *}(P \leq 0.01), \mathrm{NS}=$ no significativas.

La relación hoja:tallo presentó diferencias altamente significativas $(P \leq 0.01)$ entre estaciones del año (Cuadro 2); la relación H:T promedio por estación fue 1.31 en otoño, 1.24 en invierno y 0.91 en primavera. Álvarez ${ }^{(23)}$ al evaluar diez variedades de alfalfa demostró que independientemente de la variedad, la relación fue 1.6 en otoño, 1.4 invierno, 1.1 primavera y 1.0 verano. En cambio, otros autores ${ }^{(10)}$, observaron mayor relación H:T en invierno con un valor de 1.04 y menor en primavera con 0.84 . Entonces la menor relación en primavera en éste y otros trabajos, se asocia con mayores tasas de crecimiento, provocando un acelerado recambio de tejido y mayor contribución del tallo al cociente hoja/tallo ${ }^{(26)}$. Durante esta estación, las hojas son más abundantes en la fase vegetativa y menos abundantes conforme la planta madura y florece debido a la senescencia del tejido, además los tallos se alargan y las ramas laterales se desarrollan ${ }^{(27)}$.

Se observaron diferencias significativas $(P \leq 0.05)$ en la relación hoja:tallo entre poblaciones en invierno, primavera, y otoño (Cuadro 2); San Miguel x Oaxaca y Oaxaca x San Miguel, produjeron 11 y $9 \%$ mayor relación hoja:tallo, que el promedio de las variedades progenitoras. Aunque el mejoramiento genético en alfalfa es complicado debido a su nivel de autotetraploidía y el alto nivel de polinización natural presente en la especie ${ }^{(6)}$; Brummer $^{(28)}$, sugirió desarrollar poblaciones semi-híbridas, que se originan en un cruzamiento de dos poblaciones altamente heterocigóticas, con el propósito de capturar el vigor híbrido natural, el cual se verá reflejado en ganancias en rendimiento y sus componentes.

En la relación hoja:tallo por cortes dentro de estaciones del año se detectaron diferencias altamente significativas $(P \leq 0.01)$ (Cuadro 6), observando una disminución progresiva en los valores obtenidos conforme aumentó el número de cortes. Los mayores valores promedio 
fueron 1.50 en noviembre, 1.43 enero, 1.41 septiembre y 1.23 en febrero. Otros estudios determinaron que la relación hoja:tallo presentó cambios durante el periodo de estudio y fue más alta que la media de todos los cortes (0.79) en septiembre, octubre, enero, febrero y abril; los valores altos en la H:T observados en los meses invernales, se asocian al efecto de las bajas temperaturas en el crecimiento de las plantas, al disminuir la longitud de entrenudos, la longitud total del tallo y reducir su peso seco ${ }^{(10)}$. La interacción entre la densidad de población y la etapa de madurez al corte, tienen impacto en el rendimiento de hojas y tallos, en la etapa de floración temprana, la producción de tallos y hojas es igual, mientras que en la etapa de floración tardía los tallos componen el $60 \%$ y las hojas el $40 \%$ del rendimiento total ${ }^{(29)}$.

Cuadro 6: Relación hoja:tallo (H:T) promedio por corte en las diferentes poblaciones de alfalfa

\begin{tabular}{|c|c|c|c|c|c|c|c|}
\hline \multirow[b]{2}{*}{ Cortes } & \multicolumn{4}{|c|}{ Poblaciones } & \multirow[b]{2}{*}{ Promedio } & \multirow[b]{2}{*}{ Sign. } & \multirow[b]{2}{*}{ DMS } \\
\hline & San Miguel & Oaxaca & $\begin{array}{c}\text { San Miguel } \\
\text { x Oaxaca }\end{array}$ & $\begin{array}{c}\text { Oaxaca x } \\
\text { San Miguel }\end{array}$ & & & \\
\hline 03/Sep/13 & $1.43 \mathrm{Aab}$ & $1.32 \mathrm{Bb}$ & $1.51 \mathrm{ABa}$ & $1.38 \mathrm{Bab}$ & $1.41 \mathrm{~A}$ & * & 0.14 \\
\hline $29 / 0 c t / 13$ & $0.94 \mathrm{CDc}$ & $0.88 \mathrm{Ec}$ & $1.17 \mathrm{Da}$ & $1.04 \mathrm{Cb}$ & $1.01 \mathrm{C}$ & * & 0.08 \\
\hline 28/Nov/13 & $1.48 \mathrm{Ab}$ & $1.39 \mathrm{Bb}$ & $1.45 \mathrm{Bb}$ & $1.67 \mathrm{Aa}$ & $1.50 \mathrm{~A}$ & * & 0.11 \\
\hline 06/Ene/14 & $1.21 \mathrm{Bc}$ & $1.47 \mathrm{Ab}$ & $1.59 \mathrm{Aa}$ & $1.43 \mathrm{Bb}$ & $1.43 \mathrm{~A}$ & * & 0.07 \\
\hline 13/Feb/14 & 1.15 Bc & $1.24 \mathrm{Cb}$ & $1.29 \mathrm{Cb}$ & $1.43 \mathrm{Ba}$ & $1.28 \mathrm{~B}$ & * & 0.05 \\
\hline 20/Mar/14 & $0.97 \mathrm{Cb}$ & $0.97 \mathrm{Db}$ & $1.08 \mathrm{Ea}$ & $1.08 \mathrm{Ca}$ & $1.02 \mathrm{C}$ & * & 0.05 \\
\hline $17 / A b r / 14$ & $0.87 \mathrm{Db}$ & $0.85 \mathrm{~Eb}$ & $0.98 \mathrm{Fa}$ & $0.96 \mathrm{Da}$ & $0.92 \mathrm{C}$ & * & 0.06 \\
\hline Promedio & $1.15 b$ & $1.16 b$ & $1.29 \mathrm{a}$ & $1.28 \mathrm{a}$ & & & \\
\hline Sign. & ** & ** & ** & ** & & & \\
\hline DMS & 0.07 & 0.08 & 0.08 & 0.06 & & & \\
\hline
\end{tabular}

Diferente literal en mayúsculas en cada columna indica diferencias significativas entre cortes $(P \leq 0.01)$; diferente literal en minúsculas en cada hilera indica diferencias significativas entre poblaciones $(P \leq 0.05)$; DMS=Diferencia mínima significativa; * $(P \leq 0.05){ }^{* *}(P \leq 0.01)$.

En la relación hoja:tallo entre poblaciones también se detectaron diferencias significativas $(P \leq 0.05)$ para los siete cortes en las estaciones del año (Cuadro 6); la población San Miguel $x$ Oaxaca presentó los mayores valores en los cortes realizados durante septiembre, octubre, enero, marzo y abril; mientras la población Oaxaca x San Miguel presentó mayor relación hoja:tallo para los cortes de noviembre, febrero, marzo y abril. Se observó que las poblaciones obtenidas mediante cruzamiento, presentaron $10 \%$ mayor relación hoja:tallo que el promedio de las poblaciones Oaxaca y San Miguel. Se ha determinado que la selección en alto rendimiento de tallo es un método eficaz, para aumentar el rendimiento de forraje en alfalfa, pero al disminuir la cantidad de hojas, decrece la digestibilidad y el contenido de proteína de la planta ${ }^{(30)}$.

Se detectaron diferencias altamente significativas $(P \leq 0.01)$ en AP entre estaciones del año; la AP promedio de las poblaciones en primavera fue $25 \%$ mayor que en otoño e invierno (Cuadro 2). La altura de planta se relaciona con el rendimiento de materia seca; a medida que 
la pradera produce plantas más altas, también produce mayor rendimiento de materia seca y sus componentes tasa de crecimiento e índice de área foliar ${ }^{(31)}$.

Las diferencias en AP entre poblaciones también fueron significativas $(P \leq 0.05)$; en primavera la variedad Oaxaca y las poblaciones San Miguel x Oaxaca y Oaxaca x San Miguel tuvieron $5 \%$ mayor altura de planta que la variedad San Miguel; no se observaron diferencias significativas entre poblaciones en otoño e invierno (Cuadro 2). La variabilidad en rendimiento de materia seca, tasa de crecimiento, índice de área foliar y altura de planta, también se ha observado entre variedades comerciales de alfalfa en condiciones de campo en el Valle de México; la variedad Júpiter produjo mayor rendimiento de materia seca, tasa de crecimiento, índice de área foliar y altura de planta que la variedad Cuf $101^{(31)}$.

Se detectaron diferencias significativas $(P \leq 0.05)$ entre poblaciones para la AP en los siete cortes realizados durante el ciclo, excepto, en los cortes de septiembre y enero (Cuadro 7). La población San Miguel registró en promedio la mayor AP promedio en los cortes de octubre y febrero, la población Oaxaca en febrero y abril, la población San Miguel x Oaxaca en marzo y abril y Oaxaca $x$ San Miguel en noviembre, febrero y abril. También, se observaron diferencias altamente significativas $(P \leq 0.01)$ en AP entre cortes; los mayores valores de AP se presentaron en los cortes de septiembre, octubre, marzo y abril, y fueron mayores que el promedio de todos los cortes $(50 \mathrm{~cm})$; las diferencias en altura de planta entre poblaciones y estaciones del año se deben a diferencias en el genotipo y el régimen de temperatura; por ej., se observó que la altura de planta al corte en verano fue $65 \mathrm{~cm}$ y en invierno fue $32 \mathrm{~cm}$ en Chapingo, México ${ }^{(32)}$. Por otro lado, la temperatura mínima nocturna en verano es más alta que en invierno (Figura 1); estas diferencias en el régimen térmico, sin duda tienen efecto en el crecimiento y acumulación de materia seca de la planta de alfalfa.

Cuadro 7: Altura promedio por corte de las diferentes poblaciones de alfalfa

\begin{tabular}{lccccccc}
\hline \multirow{2}{*}{ Cortes } & \multicolumn{9}{c}{ Poblaciones } & & & \\
\cline { 2 - 5 } & San Miguel & Oaxaca & $\begin{array}{c}\text { San Miguel } \mathbf{x} \\
\text { Oaxaca }\end{array}$ & $\begin{array}{c}\text { Oaxaca } \mathbf{x} \\
\text { San Miguel }\end{array}$ & Promedio & Sign. & DMS \\
\hline 03/Sep/13 & $53.8 \mathrm{C}$ & $53.9 \mathrm{C}$ & $53.7 \mathrm{C}$ & $52.7 \mathrm{C}$ & $53.5 \mathrm{C}$ & $\mathrm{NS}$ & 2.6 \\
$29 / \mathrm{Oct} / 13$ & $52.7 \mathrm{Ca}$ & $49.3 \mathrm{Db}$ & $49.7 \mathrm{Db}$ & $51.0 \mathrm{Cab}$ & $50.7 \mathrm{D}$ & $*$ & 2.4 \\
28/Nov/13 & $41.8 \mathrm{Dab}$ & $41.6 \mathrm{Eab}$ & $38.6 \mathrm{Fb}$ & $45.9 \mathrm{Da}$ & $42.0 \mathrm{E}$ & $*$ & 5.8 \\
06/Ene/14 & $43.1 \mathrm{D}$ & $51.7 \mathrm{D}$ & $41.7 \mathrm{E}$ & $43.6 \mathrm{DEd}$ & $42.5 \mathrm{E}$ & $\mathrm{NS}$ & 2.1 \\
13/Feb/14 & $42.4 \mathrm{Da}$ & $41.2 \mathrm{Ea}$ & $39.3 \mathrm{Fb}$ & $41.4 \mathrm{Ea}$ & $41.1 \mathrm{E}$ & $*$ & 1.7 \\
20/Mar/14 & $55.7 \mathrm{Bc}$ & $57.2 \mathrm{Bbc}$ & $60.0 \mathrm{Ba}$ & $59.2 \mathrm{Bab}$ & $58.0 \mathrm{~B}$ & $*$ & 2.1 \\
17/Abr/14 & $60.3 \mathrm{Ab}$ & $64.4 \mathrm{Aa}$ & $63.1 \mathrm{Aa}$ & $63.7 \mathrm{Aa}$ & $62.9 \mathrm{~A}$ & $*$ & 2.2 \\
Promedio & $49.9 \mathrm{ab}$ & $49.9 \mathrm{ab}$ & $49.4 \mathrm{ab}$ & $51.1 \mathrm{a}$ & & & \\
Sign, & $* *$ & $* *$ & $* *$ & $* *$ & & & \\
DMS & 1.7 & 1.8 & 1.7 & 1.9 & & & \\
\hline
\end{tabular}

Diferente literal en mayúsculas en cada columna indica diferencias significativas entre cortes $(P \leq 0.01)$; diferente literal en minúsculas en cada hilera indica diferencias significativas entre poblaciones $(P \leq 0.05)$; DMS=Diferencia mínima significativa; * $(P \leq 0.05){ }^{* *}(P \leq 0.01)$, NS= no significativas. 
En el Cuadro 2 se presenta el número de tallos por planta en estaciones del año; a excepción de otoño se detectaron diferencias significativas $(P \leq 0.05)$ entre poblaciones en invierno y primavera. La población San Miguel presentó mayor número de tallos en invierno y primavera que Oaxaca y las poblaciones Oaxaca x San Miguel y San Miguel x Oaxaca. También, se detectaron diferencias altamente significativas $(P \leq 0.01)$ en el NT entre estaciones; el número de tallos promedio en primavera fue 9 y 4 tallos mayor que otoño e invierno, respectivamente. Se ha reportado que el diámetro del tallo de cada individuo de la población es altamente hereditario y controlado por los efectos genéticos aditivos, sugiriendo que la selección para mayor número y diámetro de tallos en alfalfa es factible, para aumentar el rendimiento ${ }^{(33)}$.

En NT en los siete cortes se detectaron diferencias significativas $(P \leq 0.05)$ entre poblaciones en los cortes de febrero, marzo y abril, y no se presentaron diferencias significativas en septiembre, octubre, noviembre y diciembre (Cuadro 8). La población San Miguel registró el mayor NT promedio en los cortes de febrero, marzo y abril, la población Oaxaca y las poblaciones derivadas por cruzamiento, presentaron menor NT en los cortes de febrero, marzo y abril, siendo iguales estadísticamente. También, se observaron diferencias altamente significativas $(P \leq 0.01)$ en NT entre cortes; los mayores valores de NT se presentaron en los cortes de noviembre, marzo y abril, y fueron mayores que el promedio de todos los cortes (13 tallos). La altura de corte en etapas avanzadas de maduración incrementa el rendimiento y el número de tallos por planta, al aumentar el número de tallos cerca de la superficie del suelo ${ }^{(33)}$.

Cuadro 8: Número de tallos (NT) promedio por corte de las diferentes poblaciones de alfalfa

\begin{tabular}{|c|c|c|c|c|c|c|c|}
\hline \multirow[b]{2}{*}{ Cortes } & \multicolumn{4}{|c|}{ Poblaciones } & \multirow[b]{2}{*}{ Promedio } & \multirow[b]{2}{*}{ Sign. } & \multirow[b]{2}{*}{ DMS } \\
\hline & San Miguel & Oaxaca & $\begin{array}{c}\text { San Miguel } \\
\text { x Oaxaca }\end{array}$ & $\begin{array}{c}\text { Oaxaca x } \\
\text { San Miguel }\end{array}$ & & & \\
\hline 03/Sep/13 & $8 \mathrm{E}$ & $7 \mathrm{E}$ & $7 \mathrm{E}$ & $8 \mathrm{E}$ & $8 \mathrm{E}$ & NS & 0.8 \\
\hline 29/Oct/13 & $10 \mathrm{D}$ & $9 \mathrm{D}$ & $9 \mathrm{D}$ & $10 \mathrm{D}$ & $10 \mathrm{D}$ & NS & 1.4 \\
\hline 28/Nov/13 & $12 \mathrm{C}$ & $12 C$ & $13 C$ & $13 \mathrm{C}$ & $13 \mathrm{C}$ & NS & 1.6 \\
\hline 06/Ene/14 & $13 C$ & $12 C$ & $12 \mathrm{C}$ & $12 \mathrm{C}$ & $12 \mathrm{C}$ & NS & 1.4 \\
\hline 13/Feb/14 & $19 \mathrm{Ba}$ & $15 \mathrm{Bb}$ & $15 \mathrm{Bb}$ & $16 \mathrm{Bb}$ & $16 \mathrm{~B}$ & $*$ & 1.7 \\
\hline 20/Mar/14 & $19 \mathrm{Ba}$ & $15 \mathrm{Bb}$ & $15 \mathrm{Bb}$ & $16 \mathrm{Bb}$ & $16 \mathrm{~B}$ & * & 1.6 \\
\hline 17/Abr/14 & $23 \mathrm{Aa}$ & $17 \mathrm{Ac}$ & $20 A b$ & $17 \mathrm{Ac}$ & $19 \mathrm{~A}$ & * & 2.0 \\
\hline Promedio & $15 a$ & $12 \mathrm{c}$ & $13 b$ & $13 b$ & & & \\
\hline Sign. & $* *$ & $* *$ & $* *$ & $* *$ & & & \\
\hline DMS & 1.1 & 1.0 & 0.9 & 0.9 & & & \\
\hline
\end{tabular}

Diferente literal en mayúsculas en cada columna indica diferencias significativas entre cortes $(P \leq 0.01)$; diferente literal en minúsculas en cada hilera indica diferencias significativas entre poblaciones $(P \leq 0.05)$; DMS=Diferencia mínima significativa; * $(P \leq 0.05){ }^{* *}(P \leq 0.01), \mathrm{NS}=$ no significativas. 


\section{Conclusiones e implicaciones}

El comportamiento productivo de las cuatro poblaciones de alfalfa estuvo influenciado por las condiciones ambientales; el rendimiento de materia seca, tasa absoluta de crecimiento, eficiencia en el uso de la radiación y número de tallos fue mayor en primavera (cortes en marzo y abril) que en invierno (cortes en diciembre, enero y febrero) y otoño (cortes en septiembre, octubre y noviembre); la relación hoja:tallo fue mayor en otoño que en invierno y primavera. Se determinó también que las familias de medios hermanos derivadas de las poblaciones San Miguel x Oaxaca y Oaxaca x San Miguel, mostraron en promedio $10 \%$ mayor rendimiento de materia seca, tasa absoluta de crecimiento y relación hoja:tallo que las familias derivadas de las poblaciones parentales San Miguel y Oaxaca; estas familias podrían utilizarse en un compuesto balanceado y recombinado genéticamente, para obtener una variedad sintética que podría recomendarse para condiciones similares a las que se tuvieron durante el presente estudio.

\section{- Literatura citada:}

1. Putnam DH, Summers CG, Orloff SB. Alfalfa production systems in California. In: Putnam DH, Summers CG editors. Irrigated alfalfa management for Mediterranean and Desert zones. Oakland, California, USA: ANR Publication; 2007:1-19.

2. Pietsch G, Friedel JK, Freyer B. Lucerne management in an organic farming system under dry site conditions. Field Crop Res 2007;102(2):104-118.

3. FAOSTAT. The Statistics Division of Food and Agriculture Organization of the United Nations. Available: http://faostat.fao.org/. Accessed Apr 10, 2015.

4. SIAP. Servicio de Información Agroalimentaria y Pesquera. http://www.siap.sagarpa.gob.mx/. Consultado 10 Abr, 2015.

5. Li X, Brummer EC. Applied genetics and genomics in alfalfa breeding. Agronomy 2012;2(1):40-61. 
6. Alarcón B, Venegas MR, Cervantes T. Manual para la producción de semilla de alfalfa en el Valle del Mezquital, Hidalgo. México: Fundación Hidalgo Produce AC. 2012.

7. Moghaddam A, Raza A, Vollmann J, Ardakani MR, Wanek W, Gollner G, Friedel JK. Biological nitrogen fixation and biomass production stability in alfalfa (Medicago sativa L.) genotypes under organic management conditions. Biol Agric Hort 2015;31(3):177192.

8. Brown HE, Moot DJ, Teixeira EI. The components of lucerne (Medicago sativa) leaf area index respond to temperature and photoperiod in a temperate environment. Europ J Agron 2005;23(4):348-358.

9. Ventroni LM, Volenec JJ, Cangiano CA. Fall dormancy and cutting frequency impact on alfalfa yield and yield components. Field Crops Res 2010;119(2-3):252-259.

10. Rivas JMA, López CC, Hernández GA, Pérez PJ. Efecto de tres regímenes de cosecha en el comportamiento productivo de cinco variedades comerciales de alfalfa (Medicago sativa L.). Téc Pecu Méx 2004;42(2):145-158.

11. García E. Modificaciones al sistema de clasificación climática de Köppen. $5^{\mathrm{a}}$ ed. México, DF: Instituto de Geografía-UNAM; 2004.

12. Spiers GA, WB McGill. Effects of phosphorus addition and energy supply on acid phosphatase production and activity in soils. Soil Biol Biochem 1979;11:3-8.

13. Lamb JFS, Sheaffer CC, Rhodes LH, Sulc RM, Undersander DJ, Brummer EC. Five decades of alfalfa cultivar improvement: Impact in forage yield, persistence, and nutritive value. Crop Sci 2006;46(2):902-909.

14. Undersander D, Hall MH, Vassalotti $\mathrm{P}$, Cosgrove D. Alfalfa germination \& growth. Madison, Wl: Cooperative Extension Publishing; 2011.

15. Hodgson JG. Grazing management: Science into practice Harlow. England: Longman Scientific \& Technical; 1990.

16. Milić D, Katić S, Boćanski J, Karagić Đ, Mikić A, Vasiljević S. Importance of progeny testing in alfalfa breeding (Medicago sativa L.). Genetika 2010;42(3):485-492.

17. Sharratt BS, Baker DG, Sheaffer CC. Climatic effect on alfalfa dry matter production: Part I. Spring Harvest. Agric Forest Meteor 1986;37(2):123-131.

18. Brown RH, Pearce RB, Wolf DD, Blaser RE. Energy accumulation and utilization. In: Hanson CH editor. Alfalfa science and technology. Madison, Wl: American Society of Agronomy; 1972:143-166. 
19. Dudley JW, Busbice TH, Levings CS. Estimates of genetic variance 'Cherokee' alfalfa (Medicago sativa L.). Crop Sci 1969;9(2):228-231.

20. Milić D, Mihailović V, Karagić Đ, Vasiljević S, Mikić A, Katić S. Efficacy of progeny tests in alfalfa (Medicago sativa L.) breeding for yield and quality. Field \& vegetable crops. Research/Ratarstvo i povrtarstvo 2011;48(2):327-332.

21. Warren WJ. Analysis of growth, photosynthesis and light interception for single plants and stands. Ann Bot 1981;48(4):507-512.

22. Villegas AJ, Hernández GA, Pérez PJ, López CC, Herrera HJG, Enríquez QJF, et al. Patrones estacionales de crecimiento de dos variedades de alfalfa (Medicago sativa L.). Téc Pecu Méx 2004;42(2):145-158.

23. Álvarez VP. Evaluación cuantitativa de diez variedades de alfalfa (Medicago sativa L.) [tesis maestría]. Montecillo, Texcoco, Edo. de Mexico: Colegio de Postgraduados; 2013.

24. Teixeira EI, Moot DJ, Mickelbart MV. Seasonal patterns of root C and N reserves of lucerne crops (Medicago sativa L.) grown in a temperate climate were affected by defoliation regime. Europ J Agron 2007;26(1):10-20.

25. Brown HE, Moot DJ, Teixeira EI. Radiation use efficiency and biomass partitioning of lucerne (Medicago sativa) in a temperate climate. Europ J Agron 2006;25(4):319-327.

26. Hernández GA, Matthew C, Hodgson J. Effect of spring grazing management on perennial rye grass-white clover pastures. 2. Tiller and growing point densities and population dynamics. NZ J Agric Res 1997;40(1):37-50.

27. Sheaffer CC, Martin NP, Lamb JFS, Cuomo GR, Jewett JG, Quering SR. Leaf and stem properties of alfalfa entries. Agron J 2000;92(4):733-739.

28. Brummer EC. Capturing heterosis in forage crop cultivar development. Crop Sci 1999;39(4): 943-954.

29. Kilcher MR, Heinrichs DH. Contribution of stems and leaves to the yield and nutrient level of irrigated alfalfa at different stages of development. Can J Plant Sci 1974;54(4):739-742.

30. Volenec JJ, Cherney JH, Johnson KD. Yield components, plant morphology, and forage quality of alfalfa as influenced by plant population. Crop Sci 1987;27(2):321-326.

31. Rojas GAR, Hernández GA, Joaquín CS, Maldonado PMA, Mendoza PSI, Álvarez VP, Joaquín TBM. Comportamiento productivo de cinco variedades de alfalfa. Rev Mex Cienc Agric 2016;7(8):1855-1866. 
32. Ramos SA, Hernández XE. Ecología de la alfalfa en México. COTECOCA y Colegio de Postgraduados. Chapingo, México. 1970.

33. Márquez-Ortiz JJ, Lamb JFS, Johnson LD, Barnes DK, Stucker EE. Heritibility of crown traits in alfalfa. Crop Sci 1999;39(1):38-43. 